\title{
Silencing of the foot-and-mouth disease virus internal ribosomal entry site by targeting relatively conserved region among serotypes
}

\author{
Takafumi Matsui $^{1}$ - Yoshio Handa ${ }^{1} \cdot$ Takehiro Kanda $^{1,2} \cdot$ Kyoko Tsukiyama-Kohara $^{1,3}$
}

Received: 21 May 2019 / Accepted: 24 July 2019 / Published online: 31 July 2019

(c) The Author(s) 2019

\begin{abstract}
Foot-and-mouth disease (FMD) is a host-restricted disease of cloven-hoofed animals, such as cattle and pigs. There are seven major serotypes of FMD virus that exhibit high antigenic variation, making vaccine strain selection difficult. However, there is an internal ribosomal entry site (IRES) element within the 5' untranslated region of the FMD virus (FMDV) RNA genome that is relatively conserved among FMDV serotypes and could be used as a pan-serotype target for disease interventions. To determine the potential for targeting the IRES as promising drug target, we designed a short interfering RNA (siRNA) targeting a relatively conserved region in the FMDV-IRES. The siRNA affected FMDV-IRES expression but not the expression of the encephalomyocarditis virus or hepatitis $C$ virus IRES. To evaluate the effects of siRNA-mediated silencing, we established cell lines expressing a bicistronic luciferase reporter plasmid, which contained an FMDV-IRES element between the Renilla and firefly luciferase genes. The designed siRNA inhibited FMDV-IRES-mediated translation in a concentrationdependent manner. In order to sustain this inhibitory effect, we designed a short hairpin RNA (shRNA)-expressing lentiviral vector. The results showed that the lenti-shRNA vector significantly suppressed FMDV-IRES activity for up to 2 weeks in cell culture. Thus, our findings in this study provided a basis for the development of effective pan-serotype FMDV inhibitors.
\end{abstract}

Keywords Foot-and-mouth disease virus · Internal ribosomal entry site $\cdot$ Short interfering RNA $\cdot$ Short hairpin RNA · Translation

Edited by William Dundon.

Electronic supplementary material The online version of this article (https://doi.org/10.1007/s11262-019-01696-6) contains supplementary material, which is available to authorized users.

Kyoko Tsukiyama-Kohara

kkohara@vet.kagoshima-u.ac.jp

1 Transboundary Animal Disease Center, Joint Facility of Veterinary Medicine, Kagoshima University, 1-21-24 Korimoto, Kagoshima, Kagoshima 890-0065, Japan

2 Department of Molecular Virology, Graduate School of Medicine, Kyoto University, 53 Shogoin-kawahara-cho Sakyo-ku, Kyoto, Kyoto 606-8507, Japan

3 Department of Animal Hygiene, Joint Facility of Veterinary Medicine, Kagoshima University, 1-21-24 Korimoto, Kagoshima, Kagoshima 890-0065, Japan

\section{Introduction}

Foot-and-mouth disease (FMD) virus (FMDV; genus Aphthovirus, family Picornaviridae) is a positive-sense, singlestranded RNA virus that causes FMD, a highly contagious disease of cloven-hoofed animals. FMD is epidemic or sporadic in numerous countries [1], and seven serotypes of FMDV, i.e., O, A, C, Asia 1, SAT1, SAT2, and SAT3 [2], have been identified. FMDV isolates show high levels of genetic diversity [3]. Serotype $\mathrm{O}$ is most prevalent, followed by serotype A [4]. The multiple serotypes and variants make disease control difficult; indeed, antigenic differences within a serotype are so great that little or no cross-protection can be achieved between strains of the same serotype [5]. Consistent with these observations, some capsid proteins exhibit sequence variation, with VP1 varying by approximately 30-50\% among serotypes [6].

There is an internal ribosomal entry site (IRES) element within the $5^{\prime}$ untranslated region (5'UTR) of the FMDV RNA genome, and this IRES mediates the translation of viral proteins $[7,8]$. Other picornaviruses, such as 
poliovirus (PV) and encephalomyocarditis virus (EMCV), and flaviviruses, such as hepatitis $\mathrm{C}$ virus (HCV), possess virus-specific IRES elements within their 5'UTRs [9, 10]. IRES can be classified into five types, designated I (PV), II (FMDV), III (hepatitis A virus), IV (HCV-like), and V (aichivirus-like), based on the higher-order structure [11]. Most eukaryotic mRNAs are translated in a cap-dependent manner, which involves recognition of the $5^{\prime}$ cap structure by the 43 S ribosome [12]. Viral mRNA has a short $5^{\prime} \mathrm{UTR}$ ( $<100$ nucleotides) and does not contain an initiation AUG, enabling protein synthesis in a cap-dependent manner, similar to most eukaryotic mRNAs $[9,10]$. In contrast, IRESmediated translation is cap-independent $[9,10]$. The translation of eukaryotic mRNA is halted by the cleavage of eIF4G with picornavirus protease (e.g., $\mathrm{PV} 2 \mathrm{~A}^{\text {pro }}$ and FMDV $\mathrm{L}^{\text {pro }}$ ), whereas protein synthesis regulated by PV or EMCV-IRES is stimulated $[13,14]$. FMDV $\mathrm{L}^{\text {pro }}$ can enhance translation targeting all picornavirus IRES, even after the inactivation of eIF2 by phosphorylation [15]. Because the IRES region is responsible for translational control functions, its nucleotide sequence is relatively conserved among FMDV serotypes.

In this study, we developed an approach to silence FMDVIRES by targeting a relatively conserved region among the seven FMDV serotypes in order to gain basic information for the establishment of pan-serotype inhibitors.

\section{Materials and methods}

\section{Cell culture and plasmids}

The human kidney cell line (HEK293) used in this study was obtained and cultured as previously described [16].

The pRF vectors containing FMDV-IRES (serotype C) [17], EMCV-IRES, and HCV-IRES [18] were kind gifts from Dr. Hirasawa and Professor Sung-Key Jang. The pCAGGS-Neo vector was constructed using pCAG Neo (Fujifilm Wako, Tokyo, Japan) and pCAGGS vectors (cat. no. RDB08938; Riken Bank, Ibaraki, Japan). Reporter genes were excised from pCAGGS/FMDV-IRES [16] using the restriction endonucleases EcoRV (Toyobo, Osaka, Japan) and BamHI (New England Biolabs, Ipswich, MA, USA). The pCAGGS-Neo/FMDV-IRES vector was generated by inserting a reporter gene into pCAGGS-Neo, which was then treated with EcoRV (Toyobo), BamHI (New England Biolabs), and rAPid Alkaline Phosphatase (Roche, Basel, Switzerland) using Mighty Mix (Takara, Shiga, Japan).

The FMDV-IRES short hairpin RNA (shRNA) expression vector was constructed using the pLL3.7 vector (cat. no. 11795; Addgene, Watertown, MA, USA). The shRNA sequence (5'-tACAGGCTAAGGATGCCCTTCA GGTAttcaagagaTACCTGAAGGGCATCCTTAGCCTG TtttttC- $3^{\prime}$, where capital letters indicate the target sequence and lower-case letters indicate the loop region) was subcloned under the $U 6$ promoter.

DNA sequencing was performed by FASMAC Co. (Kanagawa, Japan), and DNA sequence characterization was performed using GENETYX-Mac software (GENETYX Co., Tokyo, Japan) and GENBANK.

\section{Short interfering RNA (siRNA) transfection}

siRNA targeting a region of FMDV-IRES conserved among the seven serotypes (FMDV-con siRNA; Fig. 1) was designed as follows using BLOCK-iT RNAi Designer (Thermo Fisher Scientific, Waltham, MA, USA): 5'- ACA GGCUAAGGAUGCCCUUCAGGUA-3'. For control siRNA, ON-target plus siRNA control (Horizon/Dharmacon, Lafayette, CO, USA) was used.

\section{Northern blot analysis}

Total RNA was extracted using ISOGEN (NIPPON GENE Co. Tokyo, Japan) and electrophoresed in formaldehyde agarose (1.2\%) gels. Dicistronic mRNA was detected with labeled RNA using a $P v u I I$-digested $\mathrm{pRF}$ vector transcribed with T7 RNA polymerase (Renilla luciferase region) and detected with digoxigenin (DIF Northern Starter kit; Merk, Darmastadt, Germany).

\section{Transfection and lentiviral infection}

Plasmid transfection was performed using Lipofectamine LTX reagent (Invitrogen, Carlsbad, CA, USA) according to the manufacturer's specifications after the cells reached 50-70\% confluence. For the establishment of cell lines, HEK293 cells were cultured with medium containing G418 $(300 \mu \mathrm{g} / \mathrm{mL})$ after transfection with the pCAGGS-Neo/ FMDV-IRES vector. After 3-4 weeks, G418-resistant cells were identified as colonies. siRNA $(1,5$, or $10 \mathrm{nM})$ reverse transfection was performed using Lipofectamine RNAiMAX reagent (Invitrogen) according to the manufacturer's specifications. Lentiviral packaging was performed using MISSION Lentiviral Packaging Mix (Sigma-Aldrich, St. Louis, MO, USA), and infection with lentivirus was performed according to the manufacturer's instructions. Titration of lentivirus was performed via detection of green fluorescent protein (GFP) using a fluorescence microscope (Bz-x700; Keyence, Osaka, Japan). Cell viability was measured using WST assays (Dojindo, Kumamoto, Japan) by determining the optical density at $450 \mathrm{~nm}\left(\mathrm{OD}_{450}\right)$, according to the manufacturer's instructions. Luciferase assays were performed using a Dual-Luciferase Reporter Assay System (Promega, Madison, WI, USA). Luminescence was measured with a GloMax 96 Microplate Luminometer (Promega) for $10 \mathrm{~s}$, as previously described [16]. 
FMDV-IRES. seq

FMDV-0-IRES. sea

FMDV-A VIT A IRES.seq

FMDV-Asia1-IRES. sea

FMDV-SAT1-IRES. seq

FMDV-SAT2-IRES. seq

FMDV-SAT3-IRES . seq

FMDV-IRES. seq

FMDV-O-IRES. seq

FMDV-A VIT A IRES. seq

FMDV-Asia1-IRES.seq

FMDV-SAT1-IRES. sea

FMDV-SAT2-IRES. seq

FMDV-SAT3-IRES. seq

FMDV-IRES. seq

FMDV-0-IRES. seq

FMDV-A VIT A IRES.seq

FMDV-Asia1-IRES. sea

FMDV-SAT1-IRES. seq

FMDV-SAT2-IRES . seq

FMDV-SAT3-IRES. sea

FMDV-IRES. seq

FMDV-0-IRES. seq

FMDV-A VIT A IRES.seq

FMDV-Asia1-IRES.seq

FMDV-SAT1-IRES. seq

FMDV-SAT2-IRES. se 9

FMDV-SAT3-IRES. seq

FMDV-IRES. seq

FMDV-0-IRES. sea

FMDV-A VIT A IRES. seq

FMDV-Asia1-IRES.seq

FMDV-SAT1-IRES. seq

FMDV-SAT2-IRES seq

FMDV-SAT3-IRES. seq

FMDV-IRES. seq

FMDV-0-IRES. seq

FMDV-A VIT A IRES.seq

FMDV-Asia1-IRES. sea

FMDV-SAT1-IRES. sea

FMDV-SAT2-IRES sea

FMDV-SAT3-IRES. seq

FMDV-IRES.seq

FMDV-0-IRES. seq

FMDV-A VIT A IRES.seq

FMDV-Asia1-IRES. seq

FMDV-SAT1-IRES, seq

FMDV-SAT2-IRES. seq

FMDV-SAT3-IRES seq

FMDV-IRES. seq

FMDV-0-IRES. seq

FMDV-A VIT A IRES.seq

FMDV-Asia1-IRES. sea

FMDV-SAT1-IRES. seq

FMDV-SAT2-IRES. seq

FMDV-SAT3-IRES. seq
1 CACGATCTAA--GCAGGTTTCCCCAACTGACACAAAACGTGCAACTTGAAACTCCGCCTG

1 CACGATCTAC-- GCAGGTTTCCTCAACTGACACAACTCGTGCAAACT TTAACCCCGCCTG

1 CACGATCTAC - - GCAGGTCT TCACAACTGACACAAAACGTGCAATTTGGAACCCCGCCTG

1 CACGATCTAT - -GCAGGTTTCCACAACTGACACACACCGTGCAACTTGAAACTCCGCCTG

1 CACGATGAA-- -GCAGGFAACCACAACTGACAC--CTCGTGCACTTTGCGAGATTCCGCCCG

1 CACGATCTTGACGCAGGAATC ITAGACCAACCCAACCCGTGCACCTGCAAGTTTCGCCCG

1 CACGATCACA-CGCAGGAATCTTAGACCAACAACATCCGTGCAACT GCAAGT TTCGCCCG

59 GTCTTTCCAGGTCTAGAGGG TAACACTTTGTACTGTGTTTGGCTCCACGCTCGATCCAC 59 GTCTTTCCAGGTCTAGAGGGGGAGC-T ITTGTACTGTGTTTGACTCCACGTTCGGTCTAC 59 GTCTTTCCAGGTCTAGAGGGGAGACAC TTTGTACTGTGCCTGACTCCACGC TCGGTCCAC 59 GTCTTTCCAGGTCTAGAGGGGTAACAC TTTGTACTGTGA TTGACTCCACGCTCGGTCCAC 55 GTCTTTCC GGGTCTTGAGGAACAAACTTGTGTACTGAACCCAACTCCACAACTGGTCTAC 61 GTCTTTCCGGGTCTTGAGAGACAAACAGATGTACTGAGATCAACTCCACGATTGGTCTAC 60 GTCTTTCCGGGTCT TGAGAGACAAACAGATGTACTGAGACTGACTCCACGATTGGTCTAC

119 TGGCGAGTGTTAGTAACAGCACTGTTGCTTCGTAGCGGAGCATGACGGCCGTGGGAACTC

118 TGGCGAGTGCTAGTAGTGGCAATGTTG TTTCGTAGCGGAGCATGTTGGCCGTGGGAATCC

119 CGGCGAGTGTTAGTAGCGGCACTGTTGCTTCGTAGCGGAGCATGATGGCCGCGGGATCC

119 TGGCGGGTGCGAGTAACAGGACTGTTGTTTCGTAGCGGAGCATGATGGCCGTGGGATCCC

115 TGGCGCGTGCTAGTAGCGCACCTTCAGCTTCGTAGTGGAGCATGTAGGCCGCGGGACC TC

121 TAGCGGGTACTAGTAACACCCACTTTGCTTCGTAGCGGAGCACATGÁGCGGTGGGACCTC

120 TGGCAGGTGCTAGTAACACTCACT TTGCTTCGTAGCGGAGCACA GAGCGGTGGGACCTC

179 CTCCTTGGTAACAAGGACCCACGGGGCCAAAAGCCACGCCCACACGGGCCCGTCATGTGT

178 CTCCTTGGCGACAAGGACCCGCGGGGCCGCAAGCCACGTCTTT-- GACCCAACATGTGT

179 CCCCTTGGTAACAAGAACCCGCGGGGCCGAAAGCCACGTCCTACG-GACCCATCATGTGT

179 CTCCTTGGTAACAAGGACCCACGGGGCCGAAAGCCACGTCCTAACGGACCCACCATGTGT

175 CTCCCTGGTAACAGGGACCCGCGGGGCCAAAAGCCACGTACTT--GTACCCTACATGTGT

181 CCCCATGGTAACATGGACCCACCGGGCCAAAAGCCACG-CCTCACGGCCTCATGTGTGT

180 CTCCATGGTGACA TGGACCCACCGGGCCAAAAGCCACG--CCTAACGGCCTCATGTGTGT

239 GCAACCCCAGCACGGCG ACTTTACTGCGAAACC CACTTTAAAGTGACATTGAAACTGGTA 235 GCAACCCCAGCACGGCAACCTTGCCACGAAAACCACTTCAAGGTAACACTGATACTGGTA 238 GCAACCCCAGCACGGCAACTTTGTCTCGAAACCCACTTCAAGGTGACACTGATACTGGTA 239 GCAACCCCAGCACGGCAACTTTACTGTGAAAAACACTTTAAGGT GACACTGATACTGGTA 233 GCAACCCCAGCACGGTTGAATGTTCG GGAAACACACGTTAAGGTAACACTGGGACTGGTA 239 GCAACCCCAGCACGGCAACTTGTTTG TGAAACA TACC TTAAGGTAACACTGÄGACTGGTA 238 GCAAACCCAGCACGGCAA TT GTITGTGAAACACA ITCTTAAGGTAACACTGAGACTGGTA

299 CCCACACACTGGTGACAGGCTAAGGATGCCCTTCAGGTACCCCGAGGTAACACGCGACAC 295 CTCAAATACTGGTGACAGGCTAAGGATGCCCTTCAGGTACCCCGAGGTAACACGGGACAC 298 CTCAGTCACTGGTGACAGGCTAAGGATGCCCTTCAGGTACOCCGAGGTAACACGCGACAC 299 CTCAACCACTGGTGACAGGCTAAGGATGCCCTTCAGGTACCCCGAGGTAACACGCGACAC 293 CTTGGTTTCTGGAGACAGGCTAAGGATGCCCTTCAGGTACCTCGAGGTAACA ÄGGACAC 299 CTTGATTTTCTGGAGACAGGCTAAGGATGCCCTTCAGGTACOCTGAGGTAACAAGAGACAC 298 CTCAGITTTCTGGAGACAGGCTAAGGATGCCCTTCAGGTACC CCGAGGTAACAAGAGACAC

359 TCGGGATCTGAGAAGGGGACTGGGGCTTCTA TAAAAGCGCTCGGTTTAAAAAGCTTCTAT 355 TTGGGATCTGAGAAGGGAT TGGGACTTCTACAAAAGTGCCCAATTTAAAAAGCTTCTAT 358 TCGGGATCTGAGAAGGGGATTGGGGCTTCTATAAAAGCGCCCAGTTTAAAAA ACTTCTAT 359 TCGGGATCTGAGAAGGGGATTGGGGCTTCTTTAAAAGCGCCCAGTTTAAAAAGCTTCTAT 353 TCGÄGATCTGAGAAGGGGAC CAGGGCTTCTTTAAAAGTACC TGGTTTAAAAAGCTTCTAT 359 TTCGGGATCTGAGAAGGGGACCĀGGAGTTCTATCAAACTGCCGGTTTAAAAAGCTTCTAT 358 TCGGGATCTGAGAAGGGGACCAGGAG ITCTTTAAAACTGTCTGGTTTAAAAAGCTTCTAT

419 GCCTGAATAGGTGACCGGAGGTCGGCACCTTTCCTTTACAATTAATGACCCT 415 GCCTGAATAGGTGACCGGAGGCCGGCACCTTTTCTCTACAACTACTTTACC 418 GCCTGAATAGGCGACCGGAGGCCGGCGCCTTTCATCAACTACCAATGTC 419 GCCTGAATAGGCGACCGGAGGCCGGCGCCTTTCT TTTAACCACTACTCAAC 413 GCCTGAATAGGTGACCGGAGGCCGGCACCTTTTCTTTACTCTTTTTAA 419 GCCTGGATAGGTGACCGGAGGCCGGCACCTTTTCCTTTACTTTAAA 418 GCCTGAATAGGTGACCGGAGGCCGGCACCTTTTCCTTTA
Fig. 1 Alignment of FMDV-IRES sequences from seven FMDV serotypes. Alignment of FMDV-IRES sequences from seven serotypes (GENETYX software, multiple alignment). The FMDV-IRES sequences included in this study were classified as serotypes $\mathrm{O}$ (GenBank Sequence ID: DQ478936.1), A (GenBank Sequence ID: KY322680.1), Asia 1 (GenBank Sequence ID: AY687333.1), SAT1
(GenBank Sequence ID: MF678823.1), SAT2 (GenBank Sequence ID: KY825724.1), SAT3 (GenBank Sequence ID: KM268901.1), and C (GenBank Sequence ID: AF274010.1). The highly conserved region (nucleotides 312-339) is outlined in purple. The conserved region is outlined in red 
Fig. 2 Effects of siRNA on FMDV-IRES, EMCV-IRES, and HCV-IRES. a Schematic representation of FMDV-IRES domains $2-5$ in the 5 'UTR.

The purple line represents the position of siRNA. b HEK293 cells were cotransfected with FMDV-IRES targeting siRNA and pRF-FMDV, pRF-EMCV, pRF-HCV, and pRF vectors. Firefly and Renilla luciferase activities were measured, and IRES activity was calculated as the ratio of firefly luciferase activity to Renilla luciferase activity. The ratio (\%) of the WST value $\left(\mathrm{OD}_{450}\right)$ relative to the untreated sample is shown under the diagrams

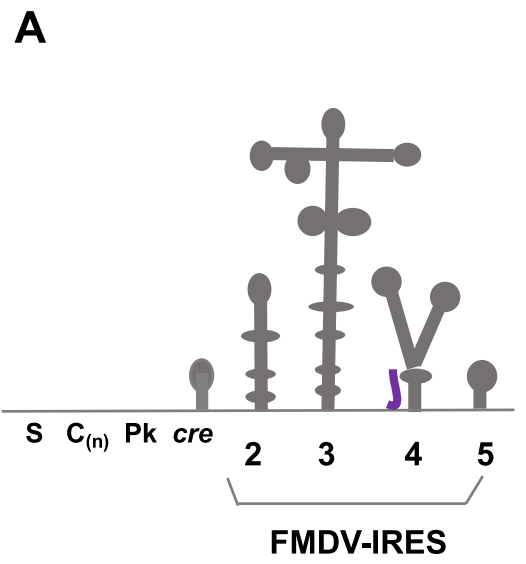

C

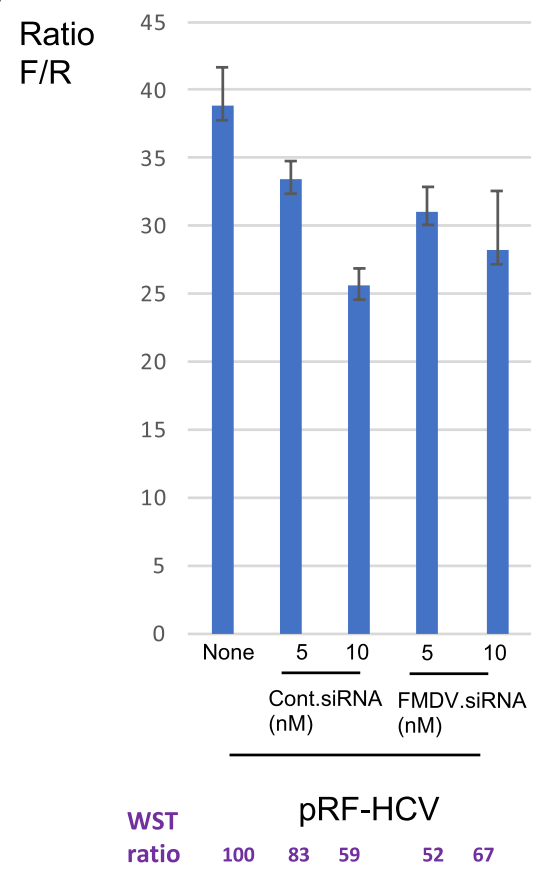

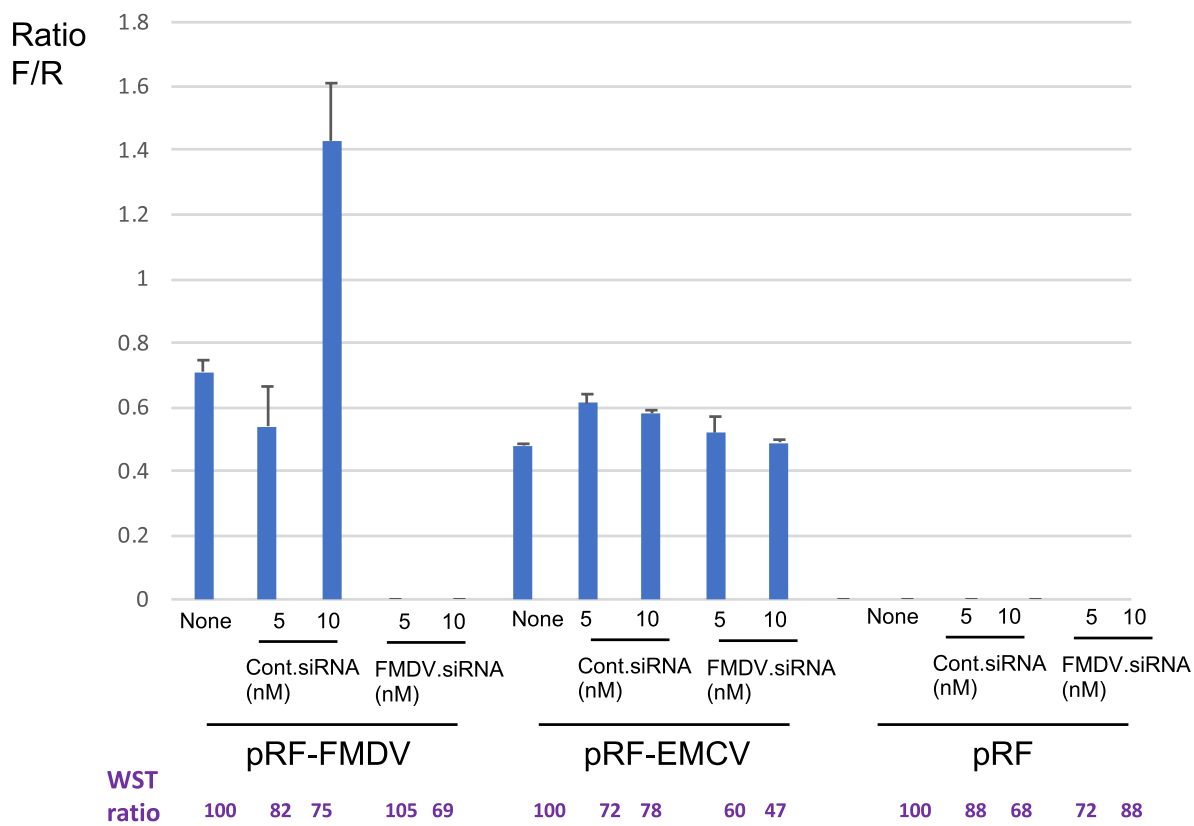

\section{Statistical analysis}

All data are presented as means \pm standard deviations from three independent experiments. Statistical analysis was performed using multiple $t$ tests corrected for multiple comparisons using the Holm-Sidak method (Graph Pad Prism ver. 8.1.2) to evaluate significant differences. Results with $P$ values of less than 0.05 were considered significant.

\section{Results}

\section{Identification of the conserved region among FMVD-IRES sequences and design of the siRNA sequence}

To identify the conserved region among FMDV-IRES sequences, we aligned the 5'UTR sequence (nucleotide 


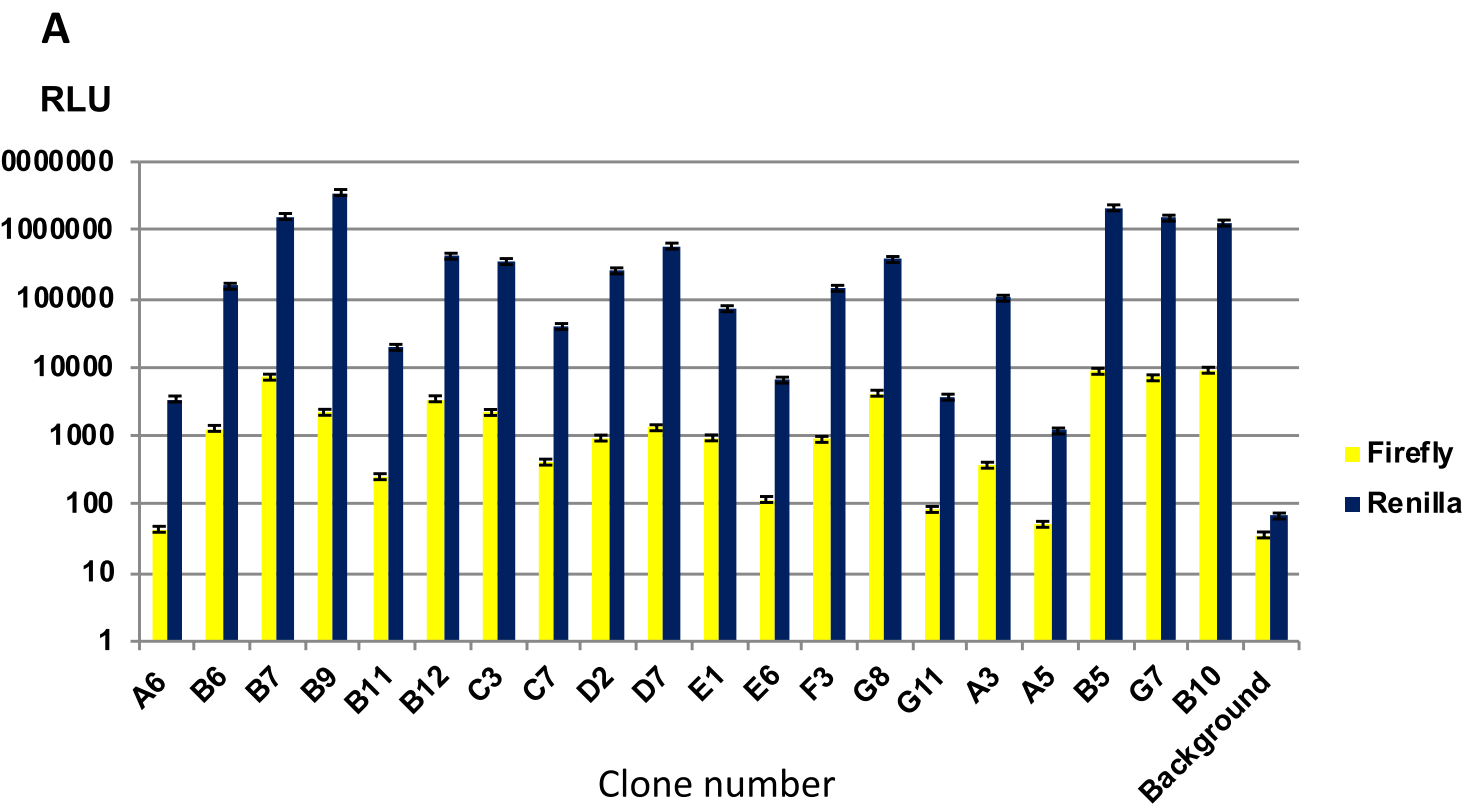

B

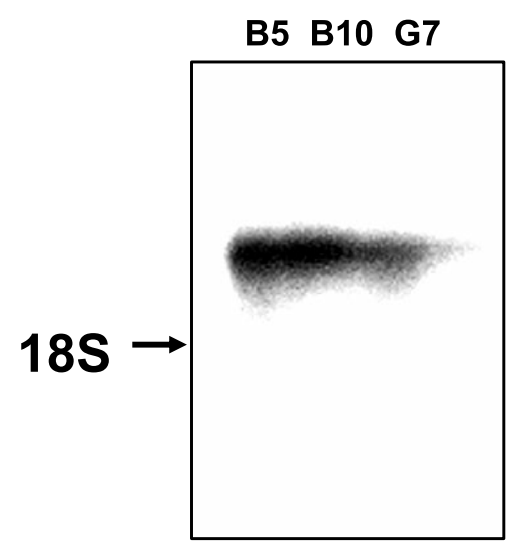

Fig. 3 Establishment of cell lines expressing bicistronic luciferase. a HEK293 cells were transfected with the pCAGGS-Neo/FMDVIRES vector. FMDV-IRES-expressing clones were established and verified by measurement of firefly (FMDV-IRES activity) and Renilla (cap-dependent translation) luciferase activities. Experiments were

numbers 569-1,038 in FMDV serotype C, AF274010.1) with representatives of each of the other six serotypes of FMDV (Fig. 1). As shown in Fig. 1 and Supplementary Fig. 1, the region encompassing nucleotide positions $312-339$ in the IRES region was highly conserved among these clones and was chosen for design of siRNA. The designed siRNA was located in the domain 4 region (Fig. 2a) [19], and the efficacy of this siRNA targeting FMDV-IRES was examined by cotransfection with the dicistronic vectors pRF-FMDVIRES, pRF-EMCV-IRES, and pRF-HCV-IRES (Fig. 2b). Our results showed that FMDV-IRES targeting siRNA could inhibit FMDV-IRES activity but not EMCV-IRES and HCVIRES activity. performed in triplicate, and error bars indicate standard deviations. b Northern blot analysis of dicistronic mRNA in cells. Total RNA ( $1 \mu \mathrm{g} /$ lane) extracted from B5, B10, and G7 cells was hybridized with the Renilla luciferase RNA probe. The position of $18 \mathrm{~S}$ rRNA $(1.9 \mathrm{~kb})$ is indicated

\section{Establishment of FMDV-IRES-expressing cell lines}

In order to evaluate the efficacy of siRNA-mediated FMDVIRES silencing, we established cell lines containing a bicistronic reporter plasmid [16]. Using EcoRV and BamHI, a fragment was excised from the pCAGGS/FMDV-IRES vector [16], which contained an FMDV-IRES element [19] between the Renilla and firefly luciferase genes. This fragment was then inserted into the pCAGGS-Neo/MCS vector. The resulting plasmid construct was named pCAGGS-Neo/ FMDV-IRES (Supplementary Fig. 2).

A previous study indicated that FMDV-IRES activity could be detected in HEK293 cells, similar to other 


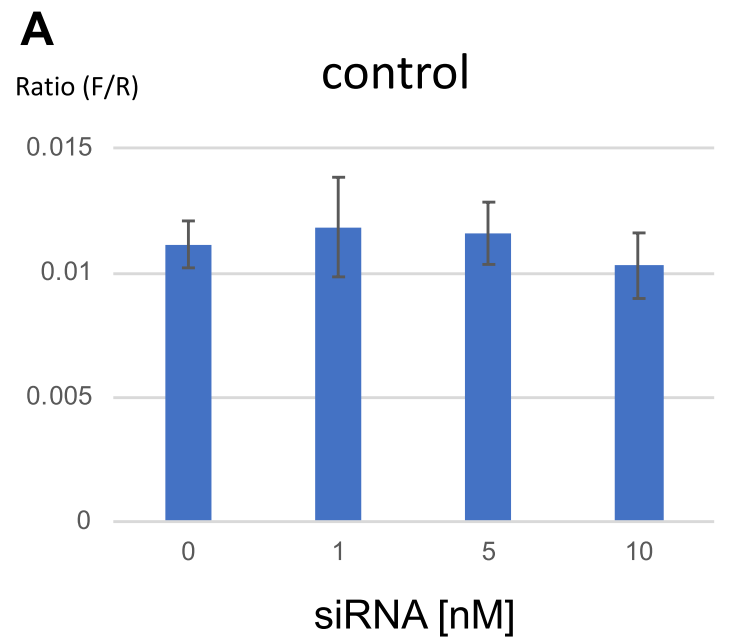

B
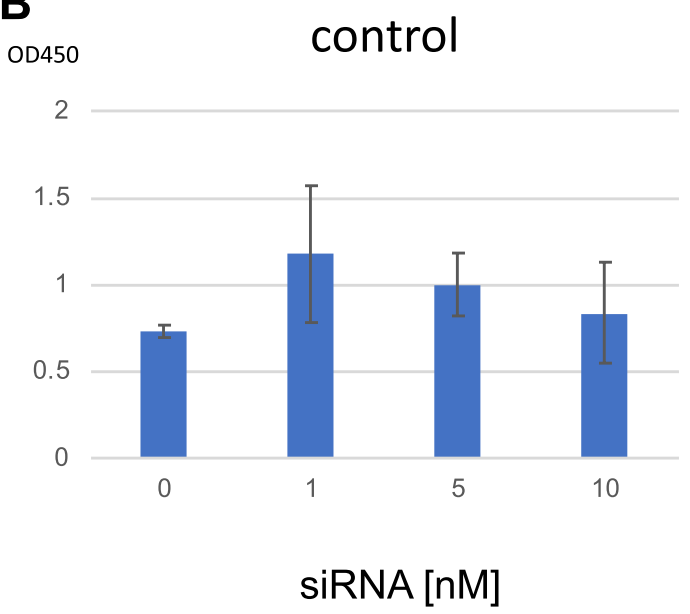

Fig. 4 Effects of siRNA on FMDV-IRES activity. a siRNA targeting the conserved region of FMDV-IRES was reverse transfected into HEK293 cells (clone B10) using Lipofectamine RNAiMAX and incubated for $48 \mathrm{~h}$. Firefly (FMDV-IRES activity) and Renilla (capdependent translation) luciferase activities were measured. To evaluate IRES-mediated translational activity, the ratio of IRES-mediated translation to cap-dependent translation was calculated. Experiments

FMDV-susceptible cell lines [16]. Therefore, the abovegenerated plasmid was then transfected into HEK293 cells and selected by treatment with neomycin. Renilla luciferase activity was measured to detect cap-dependent translation, and firefly luciferase activity was measured to detect IRES-mediated translation. We obtained 20 cell lines stably expressing Renilla and firefly luciferase, which could then be used for the screening of FMDV-IRES inhibitors (Fig. 3a). We examined the top three clones with the highest FMDV-IRES activities (B5, B10, and G7) and observed similar expression of dicistronic mRNA (3.1 kb, Fig. 3b).
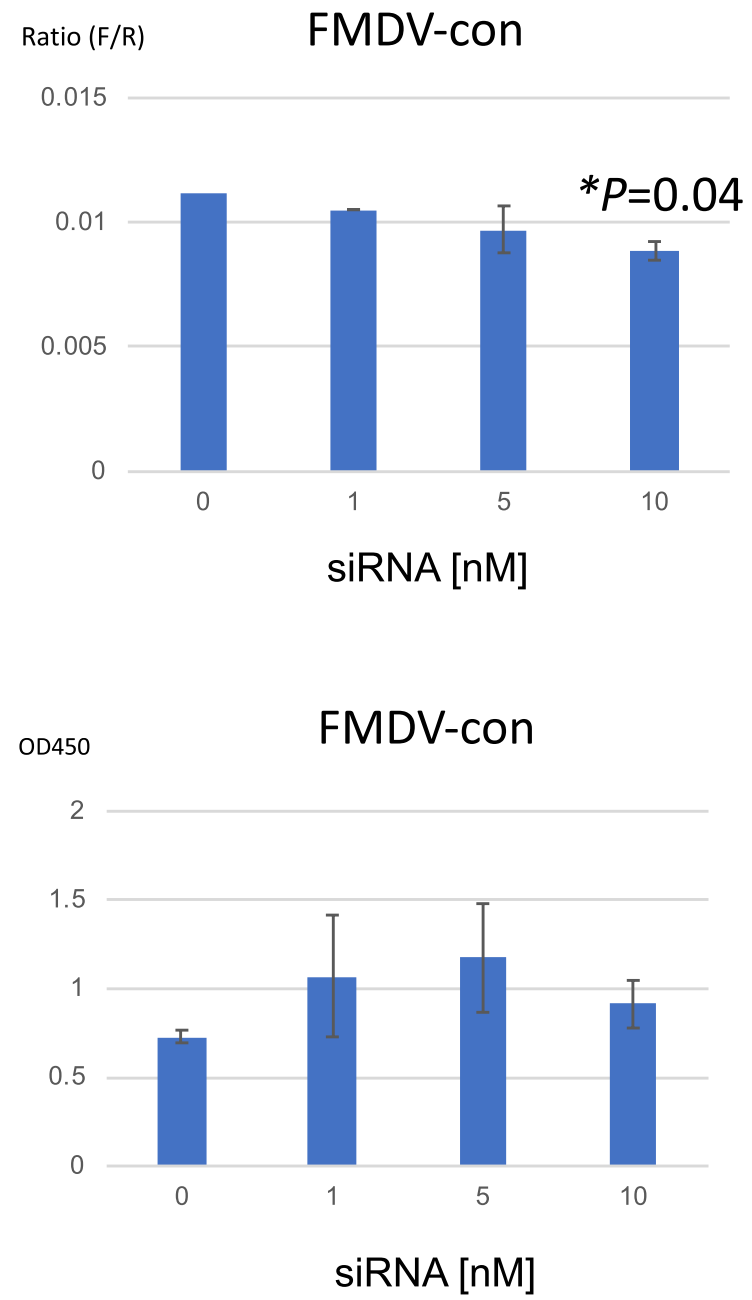

were performed in triplicate, and error bars indicate standard deviations. Multiple $t$ tests were performed to calculate $p$ values $(* p<0.05$ compared with siRNA $0 \mathrm{nM}$ ) between control cells and cells transfected with FMDV siRNA for each concentration. b Cell viability was measured using WST assays by determination of the $\mathrm{OD}_{450}$. Experiments were performed in triplicate, and error bars indicate standard deviations

From these results, we chose clone B10, which possessed the highest FMDV-IRES activity, for further characterization.

\section{Effects of siRNA on IRES-mediated translation of FMDV}

Using FMDV-IRES-expressing cells, we examined the efficacy of the designed siRNA targeting the conserved region of the FMDV-IRES sequence (FMDV-con siRNA, Fig. 4). To evaluate IRES-mediated translational activity, the ratio of IRES-mediated translation to cap-dependent translation was calculated. Treatment with FMDV-con siRNA resulted 
A

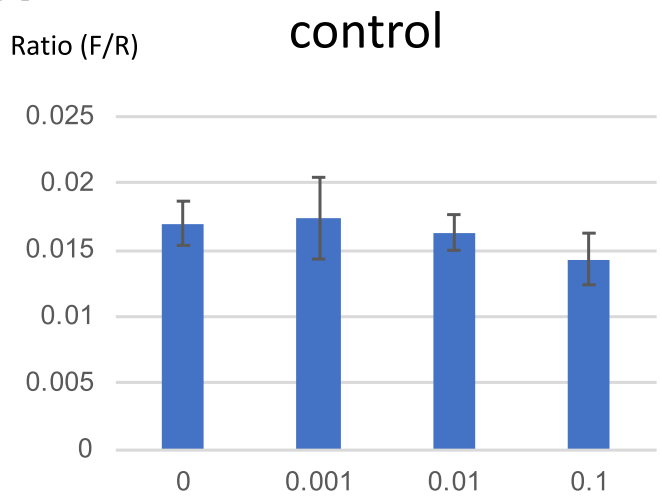

$\mathrm{MOI}$

B

OD450

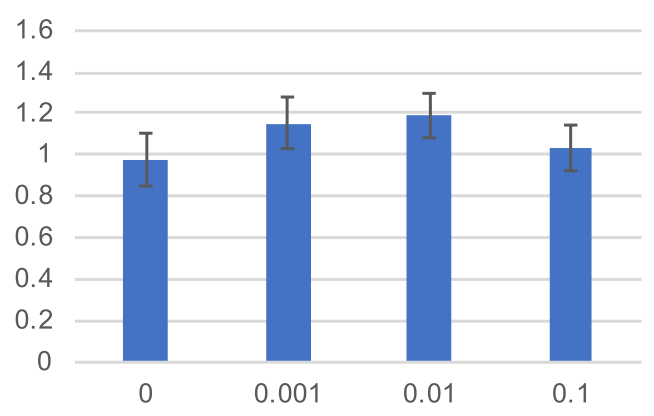

$\mathrm{MOI}$

Fig. 5 Effects of shRNA on FMDV-IRES activity. a The shRNAexpressing lentiviral vector targeting the conserved region of FMDVIRES (Lenti-FMDV-sh) or control vector (pLL3.7 alone) was used to infect HEK293 cells at various MOIs. After 14 days, firefly (FMDVIRES activity) and Renilla (cap-dependent translation) luciferase activities were measured. To evaluate IRES-mediated translational activity, the ratio of IRES-mediated translation to cap-dependent

in inhibition of FMDV-IRES activity in a concentrationdependent manner, whereas control siRNA did not show a significant effect (Fig. 4a). Moreover, treatment with the FMDV-IRES-targeted siRNA did not result in significant cytotoxicity (Fig. 4b).

\section{Establishment of an shRNA expression vector targeting the FMDV-IRES conserved region}

To sustain the effects of silencing, we constructed an shRNA expression vector utilizing a lentiviral expression vector (Supplementary Fig. 3A). We subcloned the shRNA

\section{Ratio $(F / R) \quad$ Lenti FMDV-sh}
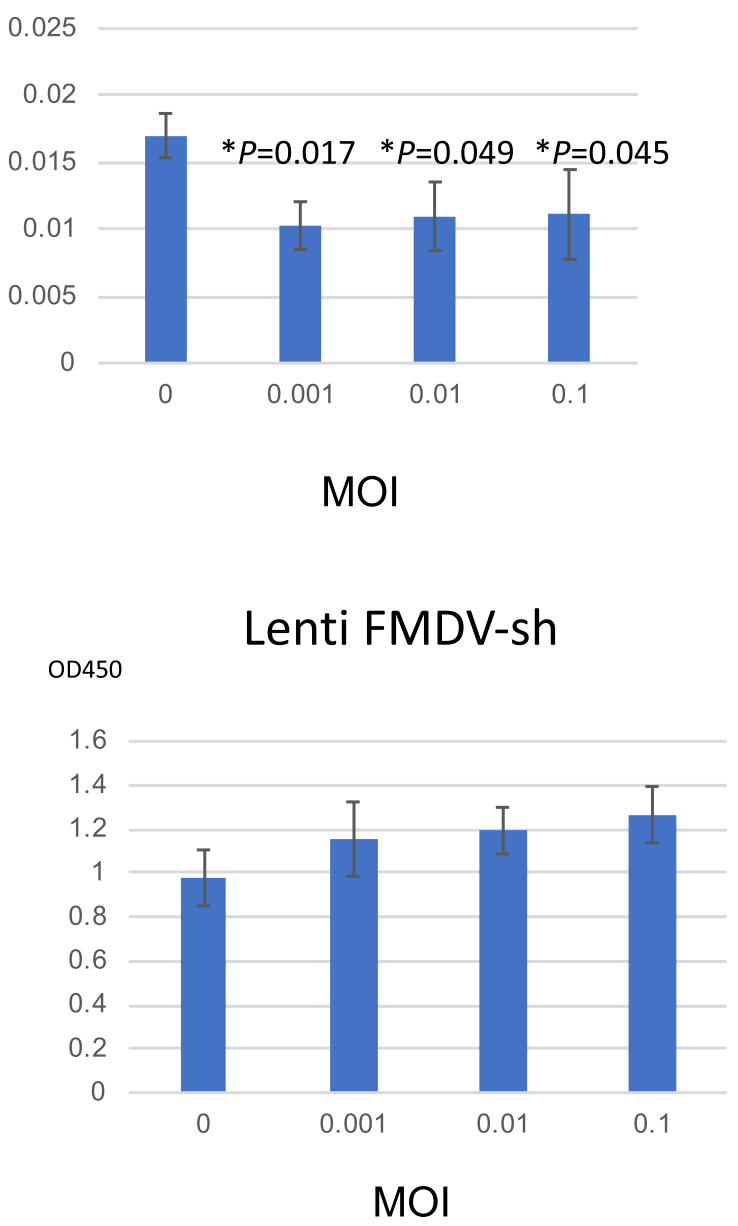

translation was calculated. Experiments were performed in triplicate, and error bars indicate standard deviations. Multiple $t$ tests were performed to calculate $p$ values between cells with and without shRNA $(* * p<0.05$ compared with $\mathrm{MOI}=0)$. b Cell viability was measured using WST assays by determining the $\mathrm{OD}_{450}$ after 14 days. Experiments were performed in triplicate, and error bars indicate standard deviations

sequence under the control of the U6 promoter. Transfection with the shRNA expression vector was confirmed with fluorescence microscopy using the GFP gene encoded in this vector (Supplementary Fig. 3A, B).

We transduced the resulting Lenti-FMDV-sh vector into cells (clone B10) and measured the IRES activity and cell viability after 14 days (Fig. 5). Transduction with the Lenti-FMDV-sh vector at a multiplicity of infection (MOI) of 0.001-0.1 significantly suppressed FMDV-IRES activity (Fig. 5a). Moreover, transduction with the Lenti-FMDV-sh vector did not significantly affect cell viability (Fig. 5b). 


\section{Discussion}

In this study, we evaluated the silencing effects of siRNA and shRNA targeting a conserved region of the FMDV-IRES among the seven FMDV serotypes. The lenti-shRNA expression vector generated shRNA under control of the U6 promoter persistently [20], enabling observation of its suppressive effects on FMDV-IRES-mediated translational activity in HEK293 cells after 14 days without significant cytotoxicity. These features make this construct promising in terms of solving several current challenges with FMDV vaccination. Moreover, this lenti-shRNA expression vector could be applicable to transduction in embryos for construction of transgenic animals [21]. For establishment of therapeutic vectors in vivo in future studies, it will be necessary to use vectors other than lentiviruses, e.g., adenovirus vectors [22, 23] and adeno-associated virus vectors [24].

FMDV populations show high levels of genetic diversity, mainly because of the lack of RNA polymerase proofreading ability. This diversity makes disease control using vaccines and laboratory diagnosis difficult [3]. For example, because of antigenic diversity between serotypes and genotypes, vaccination with another serotype or genotype of the same serotype may fail to control disease [25]. In addition, new variant viruses generated after vaccination through escape mutation can cause vaccine failure [26]. However, for the IRES element, the nucleotide sequence is relatively more conserved than that of viral structural proteins. Because IRES function is supported by higher-order RNA structures, including stem-loop structures, disruption of these structures decreases IRES activity. Thus, IRES mutants do not replicate efficiently, selecting for relative conservation of the IRES region [27]. Moreover, the IRES conserved region could be a target for prevention of FMDV infection [28, 29]. This makes the IRES region a suitable target for pan-serotype antiviral drugs for FMDV. However, there is also a risk for generating resistant escape mutant viruses [30], and this should be evaluated in future studies.

The efficacies of vaccines and drugs can be influenced by the host animal species. For example, levels of FMDV replication are significantly higher in pigs than in other animals [31], which may be related to the influence of host factors on RNA replication. However, for IRES-mediated translation, activity is not significantly influenced by the cell line origin [16], and host translation factors (e.g., eIF4E, eIF2, and eIF3) are highly conserved among animal species. Therefore, FMDV-IRES shRNA is expected to be effective in all animal species.

FMDV is a highly contagious agent that can be studied only in special facilities with highly regulated biosecurity protocols [32]. The FMDV-IRES-expressing cells established in this study could enable the screening of new inhibitors of FMDV replication in laboratories with less stringent security clearance.

In summary, the IRES-mediated translational activity of FMDV may be a suitable target for the development of pan-serotype antiviral drugs because of the relatively high sequence conservation of IRES among FMDV serotypes. The FMDV-IRES shRNA-expressing vector and FMDV-IRES-expressing cells established in this study provide new tools for the screening of anti-FMDV drugs. Future studies to evaluate antiviral effects and improve the shRNA delivery vector system are required for establishment of anti-FMDV drugs.

Acknowledgements The authors thank Dr. D. Yamane for his valuable comments on the experimental protocols. This study was supported by a grant from the Ministry of Education, Science and Culture, Japan (Grant No. 15K14781).

Author contributions TM, YH, TK, and KTK performed experiments; KTK designed the experiment and wrote the manuscript.

\section{Compliance with ethical standards}

Conflict of interest The authors declare that they have no conflict of interest.

Ethical approval This study was performed in accordance with institutional committee protocols of Kagoshima University.

Open Access This article is distributed under the terms of the Creative Commons Attribution 4.0 International License (http://creativecommons.org/licenses/by/4.0/), which permits unrestricted use, distribution, and reproduction in any medium, provided you give appropriate credit to the original author(s) and the source, provide a link to the Creative Commons license, and indicate if changes were made.

\section{References}

1. Brown F (1999) Control of foot-and-mouth disease by vaccination. Dev Biol Stand 100:131-135

2. Rweyemamu M, Roeder P, Mackay D, Sumption K, Brownlie J, Leforban Y, Valarcher JF, Knowles NJ, Saraiva V (2008) Epidemiological patterns of foot-and-mouth disease worldwide. Transbound Emerg Dis 55:57-72

3. Domingo E, Escarmis C, Baranowski E, Ruiz-Jarabo CM, Carrillo E, Nunez JI, Sobrino F (2003) Evolution of foot-and-mouth disease virus. Virus Res 91:47-63

4. Ayelet G, Soressa M, Sisay T, Belay A, Gelaye E, Jembere S, Skjerve E, Asmare K (2013) FMD virus isolates: the candidate strains for polyvalent vaccine development in Ethiopia. Acta Trop 126:244-248

5. Araujo JP Jr, Montassier HJ, Pinto AA (2002) Extensive antigenic and genetic variation among foot-and-mouth disease type A viruses isolated from the 1994 and 1995 foci in Sao Paulo, Brazil. Vet Microbiol 84:15-27

6. Knowles NJ, Samuel AR (2003) Molecular epidemiology of footand-mouth disease virus. Virus Res 91:65-80

7. Grubman MJ, Baxt B (2004) Foot-and-mouth disease. Clin Microbiol Rev 17:465-493 
8. Belsham GJ, Brangwyn JK (1990) A region of the 5' noncoding region of foot-and-mouth disease virus RNA directs efficient internal initiation of protein synthesis within cells: involvement with the role of $\mathrm{L}$ protease in translational control. J Virol 64:5389-5395

9. Jackson RJ, Kaminski A (1995) Internal initiation of translation in eukaryotes: the picornavirus paradigm and beyond. RNA 1:985-1000

10. Tsukiyama-Kohara K, Iizuka N, Kohara M, Nomoto A (1992) Internal ribosome entry site within hepatitis $\mathrm{C}$ virus RNA. J Virol 66:1476-1483

11. Lozano G, Martinez-Salas E (2015) Structural insights into viral IRES-dependent translation mechanisms. Curr Opin Virol $12: 113-120$

12. Shatkin AJ (1985) mRNA cap binding proteins: essential factors for initiating translation. Cell 40:223-224

13. Ziegler E, Borman AM, Deliat FG, Liebig HD, Jugovic D, Kean KM, Skern T, Kuechler E (1995) Picornavirus 2A proteinasemediated stimulation of internal initiation of translation is dependent on enzymatic activity and the cleavage products of cellular proteins. Virology 213:549-557

14. Borman AM, Kirchweger R, Ziegler E, Rhoads RE, Skern T, Kean KM (1997) elF4G and its proteolytic cleavage products: effect on initiation of protein synthesis from capped, uncapped, and IREScontaining mRNAs. RNA 3:186-196

15. Moral-Lopez P, Alvarez E, Redondo N, Skern T, Carrasco L (2014) L protease from foot and mouth disease virus confers eIF2independent translation for mRNAs bearing picornavirus IRES. FEBS Lett 588:4053-4059

16. Kanda T, Ozawa M, Tsukiyama-Kohara K (2016) IRES-mediated translation of foot-and-mouth disease virus (FMDV) in cultured cells derived from FMDV-susceptible and -insusceptible animals. BMC Vet Res 12:66

17. Licursi M, Komatsu Y, Pongnopparat T, Hirasawa K (2012) Promotion of viral internal ribosomal entry site-mediated translation under amino acid starvation. J Gen Virol 93:951-962

18. Paek KY, Kim CS, Park SM, Kim JH, Jang SK (2008) RNAbinding protein hnRNP D modulates internal ribosome entry site-dependent translation of hepatitis $\mathrm{C}$ virus RNA. J Virol 82:12082-12093

19. Fernandez N, Garcia-Sacristan A, Ramajo J, Briones C, MartinezSalas E (2011) Structural analysis provides insights into the modular organization of picornavirus IRES. Virology 409:251-261

20. McIntyre GJ, Fanning GC (2006) Design and cloning strategies for constructing shRNA expression vectors. BMC Biotechnol 6:1

21. Deng S, Li G, Yu K, Tian X, Wang F, Li W, Jiang W, Ji P, Han H, Fu J, Zhang X, Zhang J, Liu Y, Lian Z, Liu G (2017) RNAi combining sleeping beauty transposon system inhibits ex vivo expression of foot-and-mouth disease virus VP1 in transgenic sheep cells. Sci Rep 7:10065

22. Chen W, Liu M, Jiao Y, Yan W, Wei X, Chen J, Fei L, Liu Y, Zuo X, Yang F, Lu Y, Zheng Z (2006) Adenovirus-mediated RNA interference against foot-and-mouth disease virus infection both in vitro and in vivo. J Virol 80:3559-3566

23. Xu YF, Shen HY, Zhao MQ, Chen LJ, Li YG, Liao M, Jia JT, Lv YR, Yi L, Chen JD (2012) Adenovirus-vectored shRNAs targeted to the highly conserved regions of VP1 and 2B in tandem inhibits replication of foot-and-mouth disease virus both in vitro and in vivo. J Virol Methods 181:51-58

24. Chen PG, Sun Z (2017) AAV delivery of endothelin-1 shRNA attenuates cold-induced hypertension. Hum Gene Ther 28:190-199

25. Paton DJ, Valarcher JF, Bergmann I, Matlho OG, Zakharov VM, Palma EL, Thomson GR (2005) Selection of foot and mouth disease vaccine strains-a review. Rev Sci Tech 24:981-993

26. Mahapatra M, Statham B, Li Y, Hammond J, Paton D, Parida S (2016) Emergence of antigenic variants within serotype A FMDV in the Middle East with antigenically critical amino acid substitutions. Vaccine 34:3199-3206

27. Lozano G, Fernandez N, Martinez-Salas E (2016) Modeling three-dimensional structural motifs of viral IRES. J Mol Biol 428:767-776

28. Fajardo T Jr, Rosas MF, Sobrino F, Martinez-Salas E (2012) Exploring IRES region accessibility by interference of foot-andmouth disease virus infectivity. PLoS ONE 7:e41382

29. Vagnozzi A, Stein DA, Iversen PL, Rieder E (2007) Inhibition of foot-and-mouth disease virus infections in cell cultures with antisense morpholino oligomers. J Virol 81:11669-11680

30. Gitlin L, Stone JK, Andino R (2005) Poliovirus escape from RNA interference: short interfering RNA-target recognition and implications for therapeutic approaches. J Virol 79:1027-1035

31. Stenfeldt C, Diaz-San Segundo F, de Los Santos T, Rodriguez LL, Arzt J (2016) The pathogenesis of foot-and-mouth disease in pigs. Front Vet Sci 3:41

32. Gabbert LR, Smith JD, Neilan JG, Ferman GS, Rasmussen MV (2018) Smart card decontamination in a high-containment laboratory. Health Secur 16:244-251

Publisher's Note Springer Nature remains neutral with regard to jurisdictional claims in published maps and institutional affiliations. 